\title{
GESTIÓN DEL RIESGO EN EMPRESAS: DURACIÓN, DURACIÓN MODIFICADA, CONVEXIDAD Y DGAP APLICADOS A EMPRESAS NO FINANCIERAS
}

FIRM RISK MANAGEMENT: DURATION, MODIFIED DURATION, CONVEXITY AND DGAP APPLIED TO NO FINANCIALS FIRMS

\section{GASTÓN SILVERIO MILANESI}

Universidad Nacional del Sur

ARGENTINA

"Los cinco dedos separados son cinco unidades independientes. Ciérralos y el puño multiplica la fuerza. Ésta es la organización”. James Cash Penney 


\begin{tabular}{rr}
\hline GASTÓN SILVERIO MILANESI \\
GeSTIÓN DEL RIESGO EN EMPRESAS: DURACIÓN, DURACIÓN MODIFICADA, CONVEXIDAD Y DGAP... \\
Págs. 27 - 47. doi: http://dx.doi.org/10.30972/rfce.0192856
\end{tabular}

\title{
RESUMEN
}

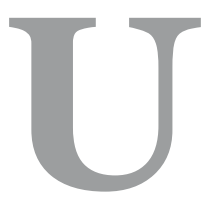

na de las principales tareas de la gerencia consiste en aplicar técnicas de administración eficiente del riesgo de la firma. El riesgo de mercado provoca cambios en el costo de capital impactando en el valor de la firma. Consecuentemente se propone adaptar un conjunto de modelos utilizados para pronosticar la sensibilidad precio ante cambios en riesgos sistémico en activos financieros: duración, duración modificada, convexidad y su implementación a empresas a partir del DGap. El trabajo se estructura de la siguiente manera: se desarrollan los conceptos correspondientes a duración, duración modificada, convexidad e inmunización. Todos ellos adaptados a la estructura de los flujos de fondos de la empresa. Luego son derivados los modelos DGap y DMGap. Con un caso de aplicación es ilustrado el funcionamiento del modelo. Además se realizó un análisis de sensibilidad para explicar la reacción de la función de convexidad y duración modificada de la empresa. Finalmente son expuestas las principales conclusiones.

Palabras clave: riesgo, duración, duración modificada, convexidad, DGap.

\begin{abstract}
One of the management's main tasks consists of applying efficient firm's risk management techniques. The market risk causes changes in the cost of capital affecting the firm value.

Consequently a whole of used models are proposed to convert in order to forecast the price sensibility in presence of changes in financial asset's systemic risk: duration, modified duration, convexity and its implementation in firms from DGap. The paper is structured as follow: it is developed the corresponding concepts to duration, modified duration, convexity and immunization. All of these adapted to the firm cash flows structure. Then they are derived the DGap and DMGap models. With an application case the model functioning is illustrated. In addition a sensibility analysis for explain the react of the firm's convexity and modified duration function is made. Finally, the main conclusions are exposed.
\end{abstract}

Keywords: risk, duration, modified duration, convexity, DGap. 


\section{INTRODUCCIÓN}

Una empresa se encuentra expuesta a un conjunto de incertidumbres operativas, financieras y de señalización e interacción con el mercado. En rigor, el grado de exposición a incertidumbre de una empresa es su riesgo y consecuentemente se debe hablar de riesgos operativos, financieros y de mercado. Ya que el riesgo tiene directa relación e impacta sobre el valor de la empresa, una de las tareas de la gerencia consiste en identificar y cuantificar la exposición al riesgo de la empresa, con el fin de tomar acciones de cobertura y por ende, reducción de la varianza de los flujos de fondos esperados. Los modelos utilizados para detectar y cuantificar riesgos son objeto de estudio de la administración del riesgo (risk management). Este es en un proceso compuesto por la identificación de los factores de riesgo operativos, financieros y de mercado; cuantificación y monitoreo a través de indicadores; seguidamente la determinación de los ratios de cobertura con instrumentos de mercado con el fin de gestionar acciones con instrumentos financieros de cobertura que permitan mitigar riesgos y maximizar el valor de la firma. Es importante destacar que el proceso de identificación y cuantificación de los factores de riesgo conduce a la toma de decisiones proactivas frente a eventos gobernables por el management y cursos estratégicos para optimizar el impacto de eventos externos no gobernables por la gerencia.

La cuantificación del riesgo y las potenciales perdidas que este puede originar es llevada a cabo mediante un conjunto de modelos. En cuanto a la estructura y comportamiento de los modelos existen dos extremos: el clásico análisis financiero en base a la información contable y modelos basados en el concepto de valor en riesgo. El primer grupo comienza con el reordenamiento de la información contable con un criterio de análisis de desempeño y estructura de financiamiento de la firma (Fornero, 2012). Emplea medidas para evaluar desempeño como el rendimiento sobre los activos operativos (ROA), costo financiero (CF), ratios de endeudamiento o composición de estructura patrimonial, rendimiento sobre el patrimonio (ROE) y efecto palanca operativos y financieros (EPO-EPF), entre otros. Se integra con el estudio de relaciones entre variables stock (activos-pasivos) y flujos (resultados) con el fin de calcular rotaciones y ciclos financieros de la firma. Los indicadores mencionados son instrumentos sencillos y básicos para el diagnóstico del riesgo de la firma. Presentan una seria debilidad, ya que en su estructura matemática no incorporan probabilidades de ocurrencia asociada a eventos externos propios del contexto ni condicionan su valor a las acciones futuras a ser implementadas por la gerencia. En síntesis, estos se constituyen en un diagnóstico financiero estático del desempeño y financiamiento correspondiente al ciclo de negocios de la empresa.

En el otro extremo se encuentran los modelos basados en el valor en riesgo (VaR, value at risk)', (Jorion, 2003), como EaR (earnings at risk), CFaR (cash flow at risk), CaR (capital at risk)

\footnotetext{
${ }^{1}$ El VaR se define como un valor límite, tal que la probabilidad de que una pérdida a precios de mercados en la cartera sobre un el horizonte temporal dado exceda ese valor, sea el nivel de probabilidad fijado. La medida se vincula a un horizonte temporal dado y suponiendo normalidad estadística en el comportamiento del retornos o precios del activo. Por ejemplo Por ejemplo, si una cartera de acciones tiene un VaR a un día de $10 \%$ sobre $\$ 1,5$ millones, existe una probabilidad del 0.10 de que la cartera caiga en valor por más de $\$ 1,5$ millones en un período de un día si no existe trading. Informalmente, una pérdida de $\$ 1,5$ millones o más en esta cartera se espera que sea de 1 día entre 20 . Una pérdida que excede el umbral del VaR se denomina "VaR break".
} 
constituyen un complemento en el análisis del riesgo. Los dos primeros se concentran en estudiar la evolución futura de las inversiones de la firma y estructura de capital a partir de las corrientes de ganancias o flujos obtenidos del estado de resultados, cuantificando valores de máxima pérdida de ganancias, para un nivel de probabilidad determinado durante un plazo temporal. Ambas medidas no estudian la evolución de los activos y pasivos de la firma ni la exposición al riesgo producto de eventos externos o de no gobierno del management. El CaR se concentra en la estructura de capital de la firma y la probabilidad de requerimientos de financiamiento extraordinarios para la cobertura de operaciones, pero no considera duraciones ni sincronización en la duración de flujos.

El presente trabajo propone adaptar a empresas no financieras, modelos empleados en activos financieros para estimar cambios en el valor ante variaciones en la tasa de interés (riesgo de mercado). Se parte del concepto de duration empleados en activos de renta fija y duration gap (DGap) utilizado en la gestión de carteras y balances de entidades financieras. En otras palabras, se desarrolla una herramienta complementaria a los modelos citados, cuyo objeto es anticipar cambios en el valor de la firma frente a variaciones en los niveles de riesgo sistémico o de mercado, considerando la estructura temporal de flujos de fondos libres (inversión) y de la deuda (pasivos) de la empresa. El punto de partida lo constituyen herramientas utilizadas en bonos como la duración $(D)$, duración modificada $(D M)$ y convexidad $(C x)$. A partir de las clásicas medidas citadas se desarrolla el concepto de duración diferencial (DGap) y duración diferencial modificada (DMGap). Adicionalmente, el modelo permite planificar acciones de cobertura, administrando las duraciones de las corrientes de flujos de fondos libres y de la deuda, con el objeto de lograr una posición de inmunización del valor de la firma.

La estructura del trabajo es la siguiente: en la primera parte son desarrollados los conceptos correspondientes a duración, duración modificada y convexidad. Seguidamente, adaptando los modelos precedentes a empresas comerciales y de servicio se deriva las ecuaciones correspondientes a DGap y DMGap, y las relaciones que subyacen entre valor y variaciones en las tasas de costo de capital. Un caso de aplicación es utilizado para ilustrar las relaciones valor - tasa de costo de capital, y la utilidad del modelo como herramienta de administración de riesgo sistémico en empresas. El caso se complementa con un análisis de sensibilidad valor activovariación tasas de costo de capital y la reacción de la curva de convexidad y duración modificada. Finalmente son expuestas las principales conclusiones.

\section{LOS MODELOS PARA MEDIR LA SENSIBILIDAD PRECIO - TASAS DE COSTOS DE CAPITAL}

En esta sección son desarrollados los modelos propuestos para gestionar el riesgo sistémico y su impacto en los niveles del costo del capital y valor. En la primera sección se intro- 
ducen las ecuaciones básicas para determinar la duración, duración modificada y convexidad de un bono; adaptadas a la lógica de la firma. Seguidamente son introducidos las ideas y conceptos de inmunización clásica, determinación del valor de la cartera en la duración y el uso de la convexidad adaptado a empresas. Finalmente son derivadas las expresiones de DGap y DMGap como herramientas para estimar y administrar la sensibilidad del valor de la firma y sus pasivos ante cambios en las tasas de costo de capital.

\section{1- DURACIÓN, DURACIÓN MODIFICADA Y CONVEXIDAD}

La duración o duration (D) en los títulos de renta fija es una medida utilizada para medir el plazo promedio de vida del activo. Representa el promedio ponderado de los diferentes vencimientos que tienen los cupones devengados por un bono. El factor de ponderación surge del pricing del bono (valor actual) y el valor actual de los flujos de pago. Consecuentemente es una medida que relaciona valor, plazo y tasa de actualización (Fabozzi, F-Fabozzi, D, 1996); (López Dumrauf, 2014). La duración de un bono de renta fija siempre es menor a su vencimiento nominal. Su expresión matemática es la siguiente,

$$
D=\frac{\partial P}{\partial Y T^{2}}=-\frac{1}{P} \times \sum_{t=1}^{\mathrm{n}} \frac{t \times C_{t}}{(1+Y)^{t}}(1)
$$

Donde $D$ representa la duración o plazo promedio, $P$ el valor actual o pricing del bono, $C_{t}$ el flujo de pago (cupón, cupón y principal o principal) y YTM la tasa de rendimiento hasta el vencimiento (yield term maturity).

La duración modificada (DM) es una medida calculada a partir de la duración, y presenta la elasticidad precio ante cambios infinitesimales en la tasa de interés. Es la primera derivada de la ecuación 1 y queda expresada como,

$$
\mathrm{DM}=-\frac{D}{(1+Y T M)}
$$

La DM tiene signo negativo atento la relación inversa entre precio y tasa de rendimiento que presentan los activos.

La convexidad $(\mathrm{Cx})$ es una medida calculada a partir de la duración. Es el complemento de la DM ya que representa la elasticidad precio ante cambios significativo en la tasa de interés, capturados en la curva precio-tasa. Por ello es la segunda derivada de la ecuación 1 y queda expresada como,

$$
C x=\frac{\partial^{2} P}{\partial Y T M^{2}}=\frac{\Sigma_{t}^{n}={ }_{1} \frac{t(1+t) \times C_{t}}{P} \times \frac{1}{(1+Y T M)^{2}}}{2}(3)
$$


Consecuentemente la variación total porcentual en el precio de un título ante un cambio en 100 puntos de la tasa de interés es capturado por DM en la recta y por Cx en la curva, actuando la última como complemento. En la siguiente ecuación se presenta la variación expresada en términos porcentuales,

$$
\frac{\partial P}{Y T M}=-D M \times \Delta y t m+C x \times(\Delta Y T M)^{2}(4)
$$

El producto entre el valor del bono y la ecuación anterior arroja como resultado la variación porcentual $\mathrm{P} \times \partial P Y Y T M$.

\section{2-ADAPTACIÓN DE LA DURACIÓN, DURACIÓN MODIFICADA Y CONVEXIDAD A LA VALUACIÓN DE EMPRESAS}

A continuación serán adaptadas las herramientas precedentes para medir las variaciones en el valor de empresas mediante el empleo de información financiera actual y proyectada. El valor intrínseco del Activo, Pasivo y Patrimonio de la empresa es estimado mediante el modelo de descuento de flujos de fondos. Con el fin de simplificar su implementación son planteados un conjunto de supuestos. En relación a la medición global del valor de la empresa se supone que los flujos de fondos libres proyectados (FCF) se comportan de manera equivalente a un bono de estructura americana, es decir, pagos de cupón a lo largo del horizonte de proyección y determinación de un valor terminal (Copeland, T -Koller, K -Murrin, J., 2000); (Pratt; SGrabowski; R, 2008); (Fernández, 2014). Los FCF se suponen asimilables a cupones y el valor terminal se supone equivalente al principal o rescate del bono. En los casos de que existan periodos donde el FCF sea negativo producto de que las inversiones incrementales proyectadas superan los flujos de fondos operativos, entonces se supone que el cupón es negativo. La tasa tomada en el modelo es el costo del capital promedio ponderado (CCPP), siendo este el sustito de la YTM en las ecuaciones precedentes. Adicionalmente se supone que las tasas de costo de capital y las estructuras objetivos (Deuda-Patrimonio Neto) se mantienen constante en el tiempo.

Bajo estas consideraciones las expresiones quedan planteadas de la siguiente manera,

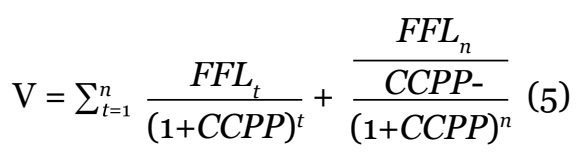

Donde $V$ el valor actual de la firma, $F F L$ flujos de fondos libres, $C C P P$ el costo del capital promedio ponderado, $n$ el final del horizonte explícito de proyección y $g$ la tasa de crecimiento (Bradley, M-Gregg, J, 2008), (Milanesi, 2017). Respecto de los pasivos la expresión queda planteada como,

$$
P=\sum_{t=1}^{n} \frac{F F L_{t}}{\left(1+k_{i}\right)^{t}}+\frac{\frac{F F L_{n}}{k_{i}}}{\left(1+k_{i}\right)^{n}}
$$


Siendo $P$ el valor actual de la deuda, $F F F$ el flujo de fondos financiero y $k_{i}$, el costo de la deuda después de impuestos. El patrimonio neto $(P N)$ puede estimarse por diferencia $(V-D)$ o directamente de aplicar el modelo de descuento de flujo de fondos,

$$
\mathrm{PN}=\sum_{t=1}^{n} \frac{F F R_{t}}{\left(1+k_{e}\right)^{t}}+\frac{\frac{F F R_{n}}{k_{e}}}{\left(1+k_{e}\right)^{n}}(7)
$$

En este caso FFR, representa el flujo de fondos residual que surge por diferencia entre flujo de fondos libres y de la deuda; $k e$ es el costo del capital propio. El mismo se puede estimar mediante modelos de equilibrio como el CAPM (Capital Assets Pricing Model) y sus derivaciones y adaptaciones a contextos emergentes (Sharpe, 1964); (Fama, E-French, K, 1992); (Elton, D- Gruber, M, 1995); (Fama, E-French, K., 1996); (Fornero, Finanzas de empresas en mercados emergentes, 2003); (Copeland, T- Weston, F- Shastri, K, 2004); (Fama, E-French, $\mathrm{K}$, 2004); (Damodaran, 2015). Los ecuaciones correspondientes a $D, D M$ y $C x$, aplicadas sobre el valor del activo de la firma $(V)$, quedan expresadas de la siguiente manera,

$$
\begin{gathered}
D_{V}=\frac{\partial V}{\partial C C P P^{2}}=-\frac{1}{V} \times \sum_{t=1}^{n} \frac{t \times F F L_{t}}{(1+C)^{t}}+\frac{n \times \frac{F F L_{t}}{C C P P-g}}{(1+C C P P)^{n}} \\
D M_{V}=-\frac{D_{V}}{(1+C C P P)}(9) \\
C x_{V}=\frac{\partial^{2} V}{\partial C C P P^{2}}=\frac{1}{(1+C C P)^{2}} \sum_{t=1}^{n} \frac{t(t+1) F F L^{t}}{(1+C C P P)^{t}}(10)
\end{gathered}
$$

Similares consideraciones son utilizadas para calcular la DP, DMP y CP correspondiente a la deuda, donde la ecuación 10 debe adaptarse a los flujos y tasa de la deuda.

\section{3-INMUNIZACIÓN}

Una estrategia básica de inmunización consiste en igualar la duración de los flujos generados por el activo con la de los pasivos, DM = DM, (López Dumrauf, 2014). Esto es posible, en tanto el comportamiento de las tasas de costo de capital del activo y pasivo sea equivalente. En el caso de que las traslaciones de las tasas no sean equivalentes no existe inmunización total, pero se puede reducir la exposición al riesgo empleando el concepto de convexidad. Por lo tanto una estrategia de inmunización debe conciliar la duración de los activos y pasivos de la empresa, con valores de continuidad post- duration que tiendan a incrementar la convexidad de los activos de la firma. 
El concepto de inmunización requiere, en primer lugar calcular el valor de la cartera a la fecha de duración. Este surge de la suma entre el valor actual de los flujos de caja anteriores a la duración y del precio de venta en ese momento. El primero surge de capitalizar los flujos anteriores reinvertidos a la tasa de mercado. El segundo surge de estimar el probable precio de venta como el valor actual de los pagos posteriores a la fecha de duración. El valor de la cartera a fecha de duración surge de la siguiente expresión,

$$
V=V A\left(F F_{D-t}\right)+V F\left(F F_{D+t}\right)(11)
$$

La expresión anterior arroja un valor similar al que se obtendría de una imposición a plazo de los flujos de fondos con vencimiento a fecha de duración, conforme surge de la siguiente ecuación,

$$
V=\Sigma_{t}^{D} F F(1+r)^{(\mathrm{D}-\mathrm{t})}+F F(1+r)^{-(D-t)}=F F(1+r)^{\mathrm{D}}(12)
$$

El concepto de inmunización, por lo tanto, se encuentra atado a las fuerzas contrarias, provocadas por los aumentos y descensos en el valor de los flujos previos y posteriores a la duración. En tal sentido, y tomando como ejemplo inversiones en activos financieros, si se desea mantener una cartera de bonos cuyos flujos estén sincronizados temporalmente con los flujos de deuda que apalancan la posición, las duraciones del activo y pasivo deben coincidir. Si la tasas de costo de capital tienden a aumentar, se incrementa el valor de la reinversión (ante duration) y baja el valor de venta (post duration). El razonamiento inverso se debe aplicar frente a un descenso de tasas.

El concepto de inmunización a ser empleado es el clásico (Fisher y Weills, 1971), siendo el valor mínimo de la cartera frente variaciones en la tasa de costo de capital. Si a una fecha determinada, en este caso fijada por la duración, la cartera de activos y pasivos generan el mismo retorno, la misma se encuentra inmunizada en su valor ante cambios en las tasas. Esto es así por la fuerza contraria de los elementos que definen el valor de una cartera a fecha de duración.

Consecuentemente, se puede inmunizar una posición larga con otra posición corta. Supóngase que la posición adeuda asciende a $N$ en un periodo $t$, tal que su valor presente es,

$$
P=N(1+k)^{-\mathrm{n}}(13)
$$

Si esta obligación es cubierta por un activo $\mathrm{V}$ con valor igual a su flujo de caja futuro se tiene,

$$
V=\sum_{t=1}^{n} \frac{F F L_{t}}{(1+k)^{t}}(14)
$$

Para estimar como queda planteado los valores del pasivo y activo, frente a cambios en la tasa de costo del capital, se debe calcular la primera derivada de cada una de las carteras; 


$$
\begin{aligned}
& P+\Delta P=P+\frac{\partial P}{\partial k} \Delta k=P+\Delta k\left[\frac{-n N}{(1+k)^{n+1}}\right] \\
& V+\Delta V \approx V+\frac{\partial V}{\partial k} \Delta k=V+\Delta k\left[\frac{-t F F L_{t}}{(1+k)^{t+1}}\right](16)
\end{aligned}
$$

Las expresiones anteriores son iguales, en el sentido de su variación, ante cambios en la tasa de costo de capital. La condición de igualdad anterior implica que un cambio en la tasa tiene un impacto neutral en los valores. Igualando ambas expresiones, simplificando y reordenando se tiene la siguiente igualdad;

$$
\frac{-t F F L_{t}}{(1+k)^{t+1}}=\frac{-n N}{(1+k)^{n+1}}(17)
$$

La expresión $\frac{N}{(1+k)^{n}}$ es igual al precio del activo $(V)$, sustituyendo en la ecuación anterior se obtiene la siguiente ecuación;

$$
\frac{1}{V} \times \frac{-t F F_{t}}{(1+k)^{t+1}}=n(17)
$$

En donde $n$ representa la duration, o sea, el horizonte de vencimiento promedio del pasivo. Si la curva de rendimientos se traslada en forma paralela, se logra inmunizar la cartera. El precio de un activo es igual al valor futuro de una obligación $N$, para cualquier cambio futuro en la tasa de interés, siempre que sus duraciones también sean iguales.

En un nivel desagregado, se puede pensar la duración del activo y pasivo de la firma como el promedio ponderado de las duraciones correspondientes a los proyectos (activos) y deudas que la integran. Si se supone divisibilidad en los activos y pasivos, condición verificable en los financieros pero no en los reales, entonces se pueden obtener duraciones objetivos trabajando las proporciones de la cartera,

$$
D_{v}=w_{1} D_{v 1}+\ldots+W_{n} D_{v n}(18)
$$

Fijando como objetivo la duración de la cartera $D_{v}$ y calculando la duración de cada uno de sus ítems $D_{v 1} \ldots . \mathrm{D}_{v n}$, se puede optimizar las proporciones que cumplen con la condición objetivo $^{2} D_{v}$.

Existen situaciones en donde los cambios en las tasas de costo del capital del activo y pasivo no son paralelos, por el contrario son arbitrarios. En ese caso, no se puede inmunizar totalmente, pero se puede reducir el riesgo. No obstante la convexidad puede servir como complemento en las estrategias de inmunización. Ya que el bono con mayor grado de convexi- 
dad frente a grandes cambios en las tasas, preserva el mayor precio, es decir le afecta menos y beneficia más un aumento o descenso en las tasas que aquel título con menor convexidad. La mayor convexidad se logra con bonos cuyos pagos se concentren sobre el final, puesto que son sensibles mayormente a cambios en las tasas (por ejemplo bonos cupón cero). Por lo general el activo es más convexo que el pasivo, dada su mayor valor terminal.

Aumentar la convexidad preservando la coincidencia en las duraciones de los activos se logra desde la cartera de activos. Para ello las proporciones y combinaciones de inversiones deben construirse fijando como fecha objetivo la duración del pasivo. Dadas las proporciones de participación de los proyectos existentes, se itera sobre el nuevo activo a ser incorporado. Para lograr un incremento en la convexidad dicho activo debe tener una estructura temporal de flujos de fondos libres muy superior a la duración objetivo ${ }^{3}$. En el caso de activos reales el efecto es generado por los valores residuales de los proyectos, por lo tanto, una empresa puede asimilarse a una corriente de flujos de fondos libres devengados por activos, coincidentes con flujos de fondos de la deuda, ambos de similar duración. El valor terminal de la empresa, posterior a la duración, tiene como efecto un incremento en la convexidad el activo.

\section{4-LA DURATION GAP (DGAP) Y LA SENSIBILIDAD VALOR - COSTO DEL CAPITAL}

Conceptualmente el DGap es la diferencia entre las duraciones correspondientes al activo y pasivo de la firma, gráficamente queda expuesto de la siguiente manera;

Duración de Activos

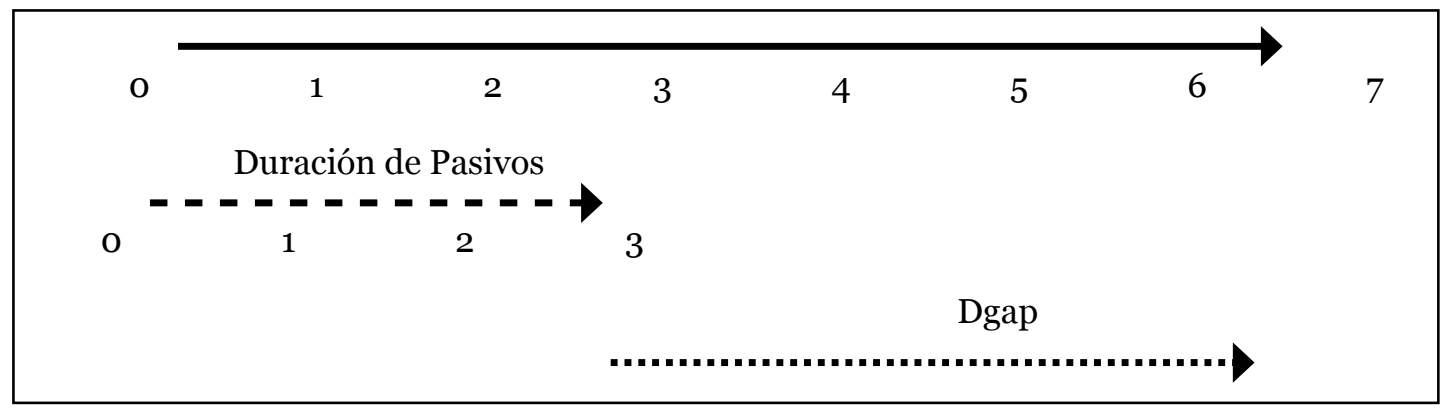

Fuente: Elaboración propia

Representa el descalce de pagos entre ingresos y fondos, donde el objetivo es la perfecta sincronización de pagos con el fin no generar capitales expuestos a las variaciones de tasas. En efecto, las relaciones entre el valor de la firma (V), variaciones en las tasas y signo del DGap se presentan en la siguiente tabla;

\footnotetext{
$\overline{{ }^{3} \text { En el caso de activos financieros la mayor convexidad es lograda incorporando un bono cupón cero de fecha superior a la }}$ duración objetivo.
} 


\begin{tabular}{|c|c|c|}
\hline DV - DP & $\Delta$ CCPP & D VALOR FIRMA \\
\hline$D_{V}>D_{P}=$ DGap $>0$ & + & - \\
\hline$D_{V}>D_{P}=$ DGap $>0$ & - & + \\
\hline$D_{V}<D_{P}=$ DGap $<0$ & + & + \\
\hline$D_{V}<D_{P}=$ DGap $<0$ & - & - \\
\hline$D_{V}=D_{P}=$ DGap $=0$ & + & NULO \\
\hline$D_{V}=D_{P}=$ DGap $=0$ & - & NULO \\
\hline
\end{tabular}

Tabla 1: Sensibilidad tasas-PN-Dgap (fuente: elaboración propia)

Cuando DGap=o implica una estrategia clásica de inmunización, donde frente a traslaciones paralelas en las tasas del activo y el pasivo se logran coberturas perfectas, producto de la coincidencias de las duraciones (ecuación 17).

Para derivar el modelo se supone que el patrimonio de la firma es sensible a las variaciones en las tasas de interés, de manera tal que: $P N\left(k_{e}\right)=V(C C P P)-\mathrm{P}\left(k_{i}\right)$ y las variaciones expresadas en cada una de los capítulos de los estados financieros sigue la siguiente lógica; $\Delta P N=\Delta V-\Delta P$.

Conforme fue planteado precedentemente, la sensibilidad del activo ante cambios en el $\mathrm{CCPP}$ es $=\partial \mathrm{V} / \partial \mathrm{CCPP} /=-\left[D_{V}(1+C C P P) /\right] \times V$ y el pasivo $\partial \mathrm{P} / \partial k_{i}=-\left[D_{p} /\left(1+k_{i}\right) /\right] \times P$.

Multiplicando denominador y numerador por $\partial C C P P, \partial k_{i}$, se obtiene la sensibilidad en el valor del patrimonio en relación a la tasa,

$$
\frac{\partial P N}{\partial C C P P}=\frac{\partial V}{\partial C C P P}-\frac{\partial P}{\partial k_{i}} \times \frac{\partial k_{i}}{\partial C C P P}(20)
$$

La cual se reduce a la siguiente expresión,

$$
\frac{\partial P N}{\partial C C P P}=\left[\frac{D_{V}}{(1+C C P P)} \times V+\frac{D_{P}}{\left(1+k_{i}\right)} \times P\right] \times \frac{\partial k_{i}}{\partial C C P P}(21)
$$

Donde $\partial k_{i} / \partial C C P P$, es la relación entre tasas de costo de capital, si estas se encuentran perfectamente correlacionadas la relación es 1 . Bajo el supuesto de que los intereses de activos y pasivos varían en similar cuantía la expresión queda planteada como,

$$
\frac{\partial P N}{\partial C C P P}=-\frac{D_{V}}{(1+C C P P)} \times V+\frac{D_{P}}{\left(1+k_{i}\right)} \times P=-\frac{V}{(1+C C P P)} \times\left[D_{V}-D_{P} \times \frac{1+C C P P}{1+k_{i}} \times \frac{P}{V}\right](22)
$$

Similares resultados son obtenidos si la derivación parte de una estrategia de inmunización de cartera, donde el cambio en el valor de los activos es igual a la variación en el valor de los pasivos (con signo contrario), $\partial V=\partial P$. En términos de duración modificada la expresión anterior puede escribirse como

$$
D M_{V} \times \mathrm{V} \times \partial C C P P=D M_{P} \times P \times \partial k_{i}(23)
$$


Despejando en función de la duración modificada de los activos, la expresión queda planteada como,

$$
D M_{V}=D M_{P} \times \frac{P \times \partial k_{i}}{V \times \partial C C P P}(24)
$$

Para obtener una duración gap de cero, se despeja sobre el lado derecho,

$$
\mathrm{O}=D M_{V}-D M_{P} \times \frac{P \times \partial k_{i}}{V \times \partial C C P P}(25)
$$

En otras palabras, la duración de los activos y pasivos es ajustada por el ratio pasivo-valor de la firma $P / V$ y el diferencial entre el costo de la deuda y CCPP $\partial k_{i} / \partial C C P P$. Al ser complejo anticipar variaciones y diferenciales de tasas, se supone que los movimientos se encuentran perfectamente correlacionados, siendo el cociente igual a 1 (Skinner, 2005). Consecuentemente la expresión queda planteada como;

$$
\operatorname{DGap}_{P N}=D_{V}-D_{P} \times \frac{P}{V},(26)
$$

Es decir el diferencial de duración ajustado por el apalancamiento, midiendo en tiempo el grado de desbalance o descalce de flujos de fondos. Mayor DGap, mayor el grado de exposición de la firma. La variación del patrimonio ante cambios en la tasa de interés es igual a,

$$
\frac{\partial P N}{\partial C C P P}=-\frac{D G a p}{1+C C P P} \times \mathrm{V}(27)
$$

Despejando en función a la variación del costo del capital el incremento en el capital como consecuencia de una modificación en tasas es igual a

$$
\partial P N=D M_{G a p} \times \partial C C P P \times V(28)
$$

La ecuación 26 cuantifica el DGap mientras que la ecuación 28 mide el cambio porcentual y en valor correspondiente al PN de la firma, como consecuencia de un cambio en una unidad porcentual del CCPP.

La expresión anterior es similar a la $D M$, medida empleada para cuantificar la sensibilidad valor frente a variaciones en la tasa del costo del capital. La duración modificada es una medida conservadora frente a la convexidad. Esto es así, debido a que pronostica, ante una variación positiva en las tasas de costo de capital, un descenso menor en el valor del activo, al pronosticado por la duración. En sentido inverso, un descenso en la tasa de interés hace que la convexidad pronostique una variación superior en el valor del activo que el informado por la 
duración modificada ${ }^{4}$. En esa línea de razonamiento el DGap es una medida aproximada y útil para pronosticar la dirección y magnitud aproximada correspondientes a las variaciones en el valor frente a cambios en el costo del capital.

\section{3-EL FUNCIONAMIENTO DEL MODELO: DGAP, DMGAP, INMUNIZACIÓN Y CONVEXIDAD DEL VALOR DE LA FIRMA}

A continuación será ilustrado el funcionamiento del DGap, DMGap y los conceptos de inmunización y convexidad mediante un caso hipotético de una empresa en marcha. Para ello supongamos una firma cuya corriente de flujos de fondos libres y de la deuda es la siguiente;

\begin{tabular}{|c|c|c|c|c|c|c|c|}
\hline Flujos de fondos & 1 & 2 & 3 & 4 & 5 & 6 & 7 \\
\hline Flujo de fondos libres & $\$ 3.747,00$ & $\$ 1.382,00$ & $\$ 1.637,00$ & $\$ 2.742,00$ & $\$ 3.550,00$ & $\$ 4.808,00$ & $\$ 73.044,00$ \\
Flujo de fondos deuda & $\$ 1.573,74$ & $\$ 580,44$ & $\$ 481,28$ & $\$ 806,15$ & $\$ 1.043,70$ & $\$-$ & $\$-$ \\
\hline
\end{tabular}

Tabla 2: Flujos de fondos proyectados de la firma (elaboración propia)

En la siguiente ilustración se expone la estructura y comportamiento de los flujos de fondos libres y de la deuda.

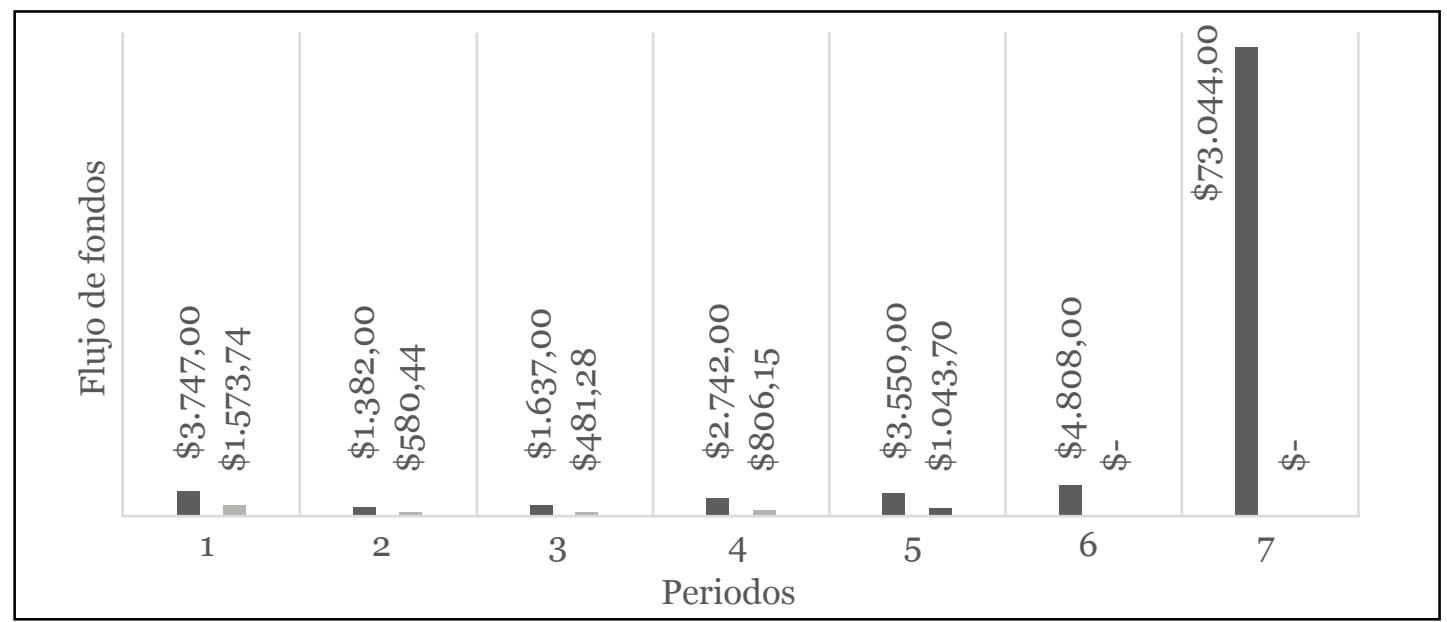

Ilustración 1: Flujo de fondos libre y de la deuda (elaboración propia)

Los pasivos de corto plazo representan el 30\% de la estructura de deuda financiera de la firma. La tasa de deuda después de impuesto, surge del costo efectivo promedio ponderado entre pasivos financieros de corto y largo plazo, siendo de $k_{i}(1-t)=8 \%$. La tasa del costo del capital propio se estimó aplicando el clásico modelo CAPM, donde el tipo sin riesgo es $r_{f}=3 \%$, el rendimiento de mercado asciende a $R_{m}=10 \%$ y el coeficiente beta es de $\beta_{i}=1,29$. La tasa de

\footnotetext{
${ }^{4} \mathrm{Si}$ se quiere incorpora la convexidad, esta debe calcularse directamente para el PN, donde los flujos de fondos libres son sustituidos por el flujo de fondos residual, a partir de la siguiente expresión $C x_{P N}=\frac{\partial^{2} P N}{\partial k_{e}^{2}}=\frac{1}{\left(1+k_{e}\right)^{2}} \sum_{t=1}^{n} \frac{t(t+1) F F R}{\left(1+k_{e}\right)^{t}}$. Por lo tanto la variación de valor queda expresada como $\frac{\partial P}{C C P P}=-D M G a p_{P N} \times \Delta C C P P+C x_{P N} \times(\triangle C C P P)^{2}$
} 
costo del capital propio es: $k_{e}=r_{f}+\left(R_{m}-r_{f}\right) \times \beta_{i}=12,07 \%$. La estructura deuda-capital propio se supone constante, siendo una firma con poco apalancamiento $(P / V=8 \% ; P N / V=92 \%)$. El costo del capital promedio ponderado (CCPP) asciende, ccpp $=w_{1} \times k_{i}(1-t)+w_{2} \times k_{e}=11,80 \%$. Con los datos de la tabla 2 y los valores correspondientes a las tasas de costo de capital se procede a calcular: valor de la firma (V), de la deuda (D) aplicando el descuento de flujos de fondos, expresado en miles de pesos (ecuaciones 5 y 6), como las duraciones correspondientes a los activos y pasivos (ecuación 8).

\begin{tabular}{|lcl|}
\hline Valor de la firma (V) & $\$ 45.335,70$ & ecuación 5 \\
Valor deuda (P) & $\$ 3.639,72$ & ecuación 6 \\
Duración Activos & 6,071 & ecuación 8 \\
Duración Pasivos & 2,616 & ecuación 8 \\
\hline
\end{tabular}

Tabla 3: Valor Firma, Deuda y duraciones (elaboración propia)

Seguidamente se calcula DGap, para ello se utiliza la ecuación 26 con el fin de obtener la medida que indica la sensibilidad precio ante un cambio unitario (100 puntos) en la tasa del costo del capital. Luego son aplicadas las ecuaciones 27 y 28, con el fin de estimar la DMGap. Estas ecuaciones son las que cuantifican cambios relativos y absolutos en el valor del patrimonio neto, ante un cambio en el tipo de interés.

\begin{tabular}{|lcc|}
\hline DGap & 5,861 & ecuación 17 \\
DM Gap & $-5,24242$ & ecuación 18 \\
$\Delta \mathrm{k}$ variación & $1 \%$ & \\
$\Delta \mathrm{k}^{*}$ DM Gap & $-5,2424 \%$ & \\
$\Delta \mathrm{k}^{*}$ DM Gap*V & $\$-2.376,69$ & ecuación 19 \\
\hline
\end{tabular}

Tabla 4: DGap, DMGap (elaboración propia)

Los resultados obtenidos en la tabla 4 indican que el DGap es de 5,86 años, siendo positivo y exponiendo la inversión en exceso de activos sobre pasivos. Este descalce de carteras, y por ende, de flujos proyectados de ingreso y egresos produce un efecto negativo ante variaciones positivas en las tasas de costo de capital. En efecto, el DM Gap asciende a -5,24 indicando que un cambio en 100 puntos en la tasa del CCPP implica una reducción del 5,24\% en el valor del Patrimonio de la firma, siendo en este caso de \$2.376,69 miles de pesos.

A continuación se presenta un análisis de sensibilidad tasa de interés valor del patrimonio aplicando las ecuaciones 8, 27 y 28 .

\begin{tabular}{|c|c|c|c|c|c|c|c|c|c|c|c|}
\hline$\Delta \mathrm{k}$ variación & $-5 \%$ & $-4 \%$ & $-2 \%$ & $-1 \%$ & $1 \%$ & $3 \%$ & $4 \%$ & $6 \%$ & $7 \%$ & $9 \%$ & $10 \%$ \\
\hline$\Delta \mathrm{k}^{*} \mathrm{DM}$ Gap*V & 11883,45 & 8318,42 & 4753,38 & 1188,35 & $-2376,69$ & $-5941,73$ & $-9506,76$ & $-13071,80$ & $-16636,83$ & $-20201,87$ & -2376 \\
\hline
\end{tabular}

Tabla 5: Sensibilidad DGap, DMGap (elaboración propia)

En efecto los valores van desde un incremento en $\$ 11.883$ miles de pesos para un descenso del $5 \%$ (una variación del $25 \%$ en el valor del patrimonio) hasta un descenso máximo de 23.766 miles de pesos, ante un aumento del 10\% en el CCPP, representando más del 53\% 
del valor de partida correspondiente al patrimonio neto. En economías emergentes, con importantes niveles de incertidumbre sistémica, la firma se encuentra expuesta a importantes variaciones de valores, en principio por movimientos en las tasas de costo de capital. En el siguiente gráfico se presenta el análisis de sensibilidad descripto.

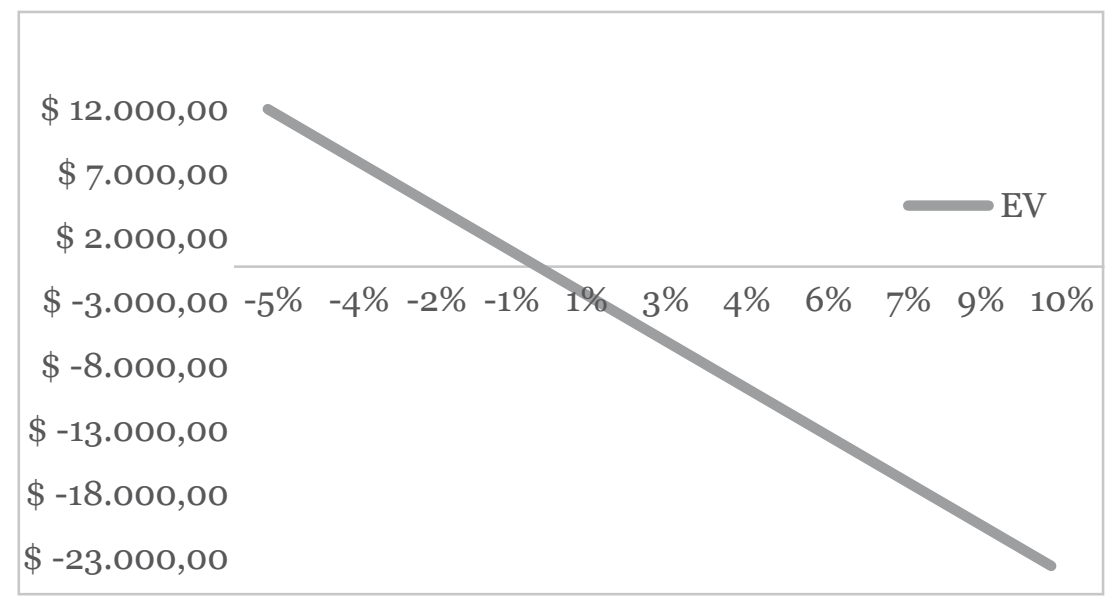

Ilustración 2: DGap (elaboración propia)

Tomando los flujos de fondos libres y aplicando la ecuación 10 se puede apreciar el efecto de la duración y convexidad sobre el activo,

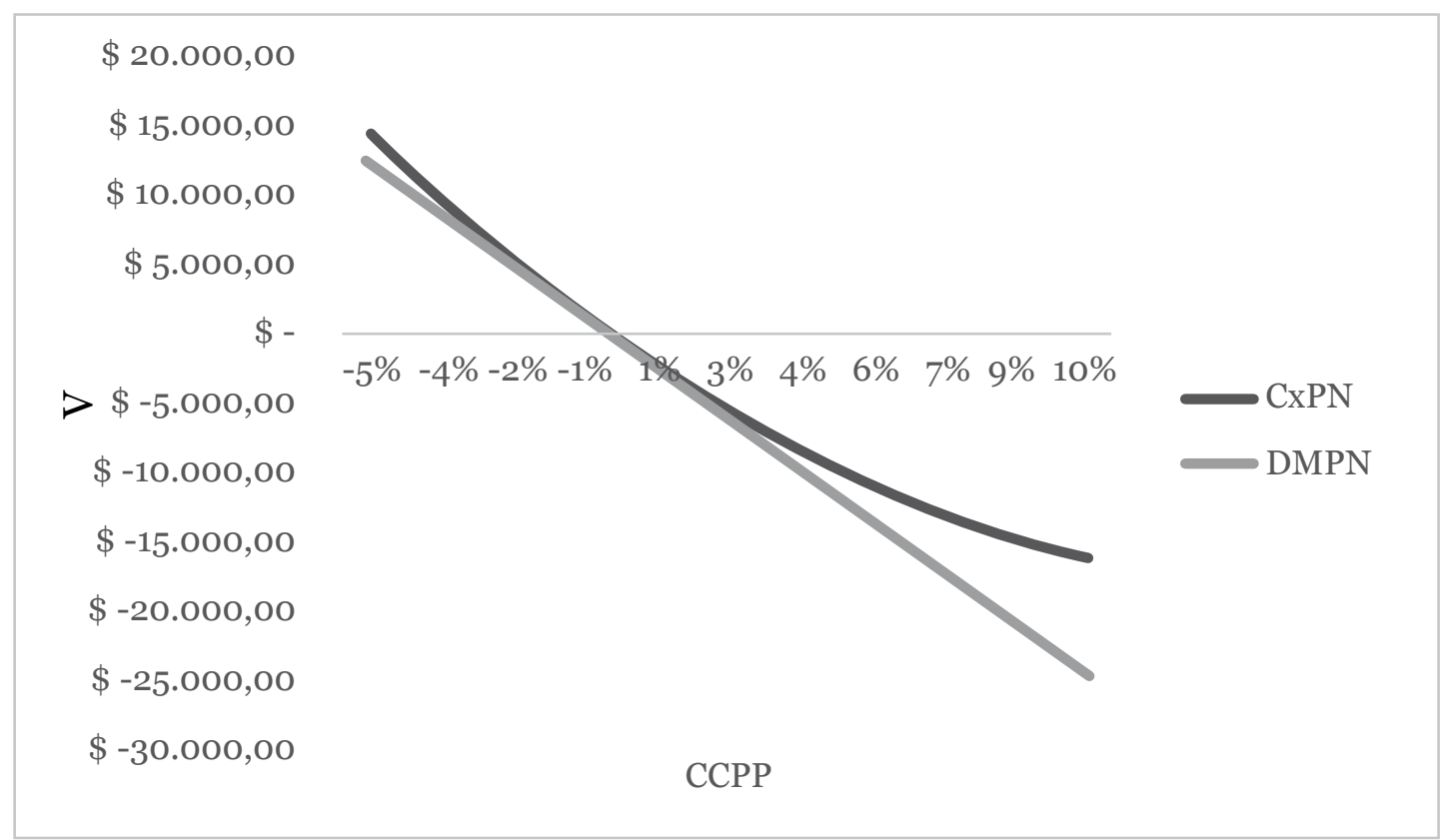

Ilustración 3: Convexidad y duración del activo frente a diferentes tasas de costo de capital (elaboración propia) 
Se verifica que la amplitud de movimientos en el valor, capturada por la DM, es mayor en el caso de pérdidas de valor (ascenso en tasas de interés) y viceversa, comparada con la convexidad (Cx). En términos prácticos, así como acontece con los activos de renta fija, se opta por trabajar con el concepto de duración ya que si bien no es una medida exacta ante variaciones significativas de tasas, arroja resultados más conservadores (amplifica pérdidas y disminuye perdidas de valor).

En el caso de plantear la inmunización del valor de la firma, esta se logra igualando las duraciones del activo y el pasivo. En este caso las acciones implicarán el aplazamiento de pagos, incrementando la duración de los pasivos o acelerando la generación temporal de los flujos de fondos libres. Estas acciones dependerán de la flexibilidad estratégica temporal de tales flujos. Igualadas las duraciones, el DGap y DMGap ascienden a cero.

Continuando con el ejemplo, se determina el valor mínimo del portfolio (VMP), adaptado al activo y el pasivo de la empresa objeto de estudio, aplicando la ecuación 12. El valor obtenido es sensibilizado tomando como variable las tasas de costo de capital, a partir de las duraciones obtenidas para activos $(6,01)$ y pasivos $(2,67)$. La tabla 6 y los gráficos 4 y 5 exponen los movimientos y sensibilidades de los flujos en relación a sus duraciones, correspondientes al activo y pasivo.

\begin{tabular}{|ccccccccc|}
\hline $5 \%$ & $7 \%$ & $10 \%$ & $12 \%$ & $13 \%$ & $16 \%$ & $18 \%$ & $22 \%$ & VA $11,8 \%$ \\
$\$ 89.791,99$ & $\$ 89.512,65$ & $\$ 89.273,59$ & $\$ 89.234,32$ & $\$ 89.251,85$ & $\$ 89.450,11$ & $\$ 89.706,35$ & $\$ 90.523,08$ & $\$ 45.335,70$ \\
$2 \%$ & $4 \%$ & $6 \%$ & $8 \%$ & $10 \%$ & $12 \%$ & $16 \%$ & $18 \%$ & VA $8 \%$ \\
$\$ 4.489,92$ & $\$ 4.497,49$ & $\$ 4.507,85$ & $\$ 4.520,88$ & $\$ 4.536,42$ & $\$ 4.554,38$ & $\$ 4.597,07$ & $\$ 4.621,62$ & $\$ 3.696,55$ \\
\hline
\end{tabular}

Tabla 6: Sensibilidad VMP flujos de fondos activo-pasivo (elaboración propia)

VA representa el valor actual correspondiente al valor final, a fecha de duración, obtenido mediante la ecuación 12, siendo este el valor de la firma y el pasivo al inicio.

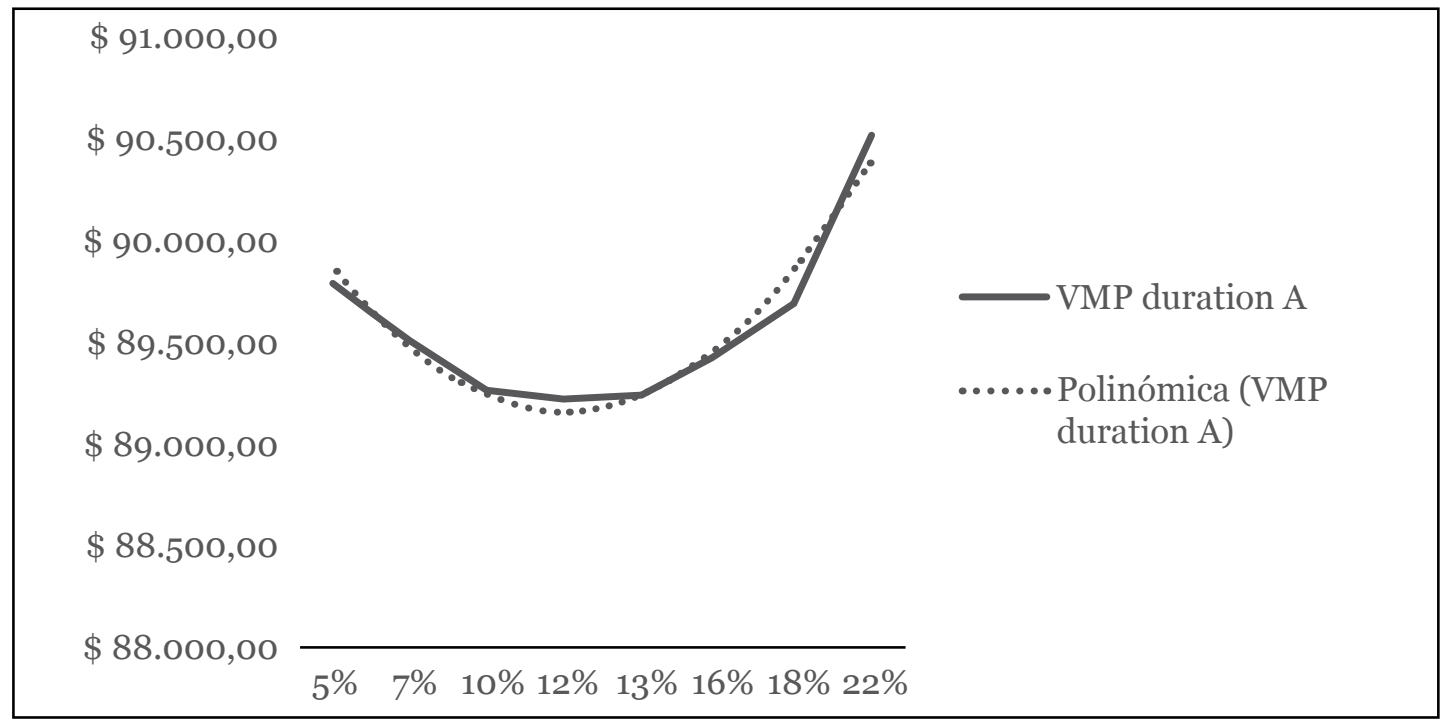

Ilustración 4: VMP del activo frente a diferentes tasas (elaboración propia) 
Se observa que el activo es más sensible que el pasivo frente a cambios en la tasa de costo de capital.

Esto es consecuencia de la mayor duración y convexidad del primero frente al segundo. Por lo tanto se advierte la posibilidad de inmunizar a las inversiones de la empresa frente a situaciones de traslaciones no simétrica en la tasas de costo de capital.

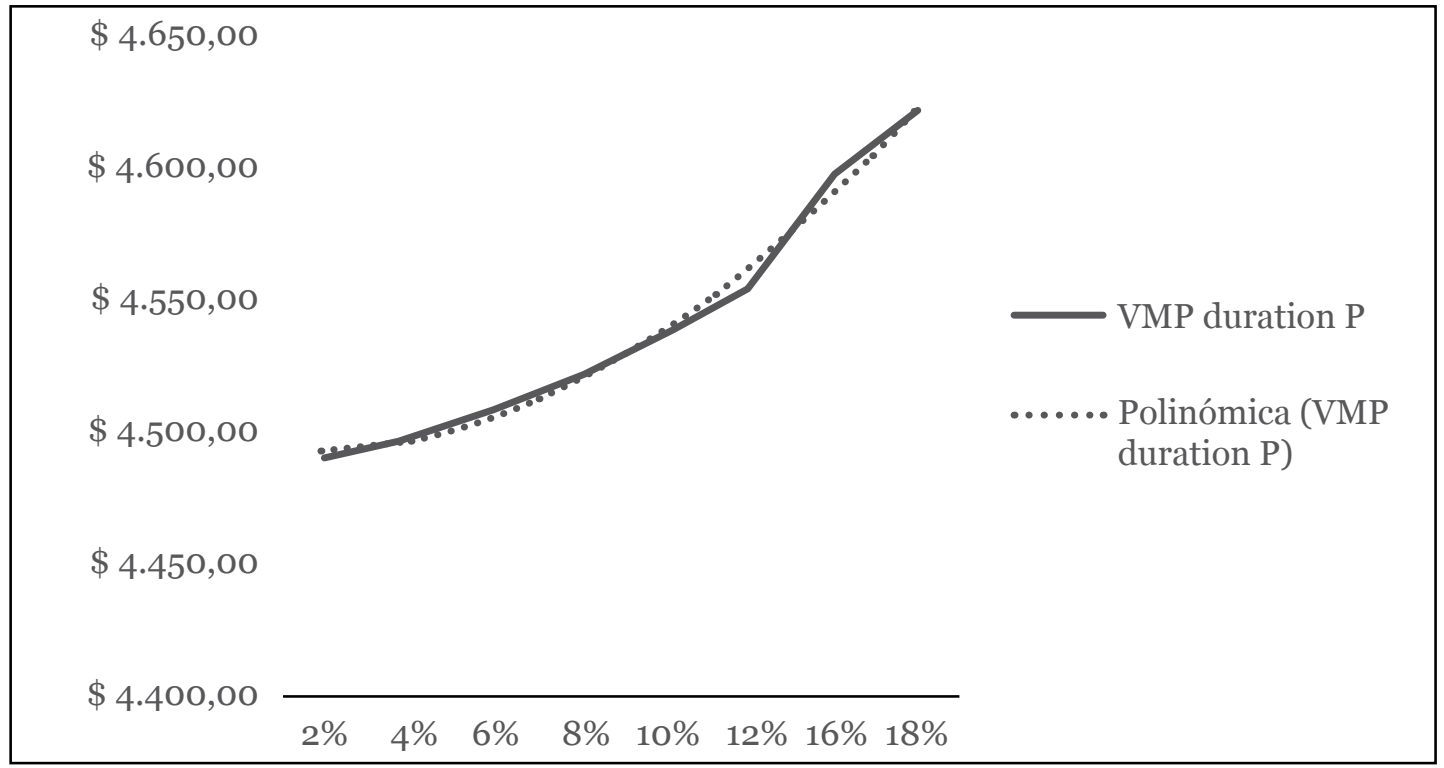

Ilustración 5: VMP del pasivo frente a diferentes tasas (elaboración propia)

En términos de convexidad los resultados quedan plasmados en la ilustración 6, donde la convexidad del pasivo es de 2,37 y el activo más del doble 5,24. En el caso de traslaciones no simétricas en las tasas de costo de capital, dicha situación es favorable para la firma. Frente a descensos en la tasa el aumento de la cartera de activos es superior al pasivo y viceversa, en casos de incremento de riesgos sistémicos o propios el ajuste negativo en el valor del activo es menor al pasivo.



llustración 6: Convexidad del activo y pasivo pasivo frente a diferentes tasas (elaboración propia) 
Conforme fue expuesto, si las traslaciones de las tasas de costo de capital son paralelas la estrategia de inmunización requiere de la coincidencia en la duración de los flujos. En otras palabras, se deben hacer coincidir las duraciones de los flujos correspondientes al activo y pasivo de la empresa. En parte, en el caso de las empresas, esto depende de la capacidad de la gerencia de postergar o adelantar flujos de deuda o o activos. Por lo general, tal flexibilidad es factible en el caso de los flujos de pasivos, ya que la estructura temporal de los flujos de las inversiones si bien son gestionables por la gerencia, a menudo queda sujetas a las características operativas, tecnológicas y de mercado del negocio. En el caso de traslaciones no paralelas de la tasas de costo de capital, se debe buscar mayor convexidad generado por una mayor duración, caso que acontece en los activos de la firma producto del impacto en los flujos del valor terminal.

\section{CONCLUSIONES}

La administración del riesgo es un proceso propio del management de la firma, donde son detectados los factores de exposición a la incertidumbre, se los cuantifica y aplica herramientas que permitan anticipar los eventos, generando las coberturas pertinentes. En esta última categoría ingresan todas aquellas situaciones que impactan directamente contra las tasas de costo del capital de la firma, modificando el valor del activo, pasivo y patrimonio. En el caso de los indicadores basados en información contable, estos no capturan tal efecto, ya que el equilibrio financiero revelado por la contabilidad es de carácter estático. Lo mismo acontece con las herramientas de la familia del VAR (value at risk), ya que cuantifican la máxima perdida posible en un intervalo de tiempo determinado. El trabajo aporta un conjunto de modelos que complementan a los precedentes, que persiguen pronosticar la sensibilidad del valor del patrimonio ante cambios en las condiciones que afectan las tasas que componen el costo del capital. En otras palabras se pretende cuantificar y anticipar la exposición al riesgo sistémico que tiene la firma y posibles acciones de inmunización. Por tal motivo se desarrolló el modelo DGap y DMGap. Es una adaptación de las herramientas utilizadas en el análisis de activos de renta fija empleada por entidades financieras, donde se analiza la sincronización existentes entre las corrientes esperadas de flujos de fondos libres y de la deuda. El modelo se basa en el concepto de duración (D) del activo y pasivo, calculada a partir del descuento de flujos de fondos (DFF) y las tasa de costo de capital promedio ponderado (CCPP), con el fin de obtener el DGap y DMGap. Conforme fue expuesto, valores positivos de DGap, propios de duraciones de activo mayor al pasivo generan exposiciones negativas de valor del patrimonio neto, frente a potenciales incrementos en el costo del capital.

Esto permite diseñar estrategias dependiendo del contexto de tasas proyectadas. En el caso de aumentos del riesgo sistémico al cual se encuentra expuesta la firma, se debe procurar que la 
duración o repago promedio del activo sea inferior a la del pasivo. La inversa es aplicable para escenarios de descenso en las tasas de costo de capital, ya que en este caso, un DGap positivo aumenta el valor del capital, producto del bajo costo de oportunidad del capital y del dinero. Paralelamente se adaptó el concepto de inmunización con el objeto de sincronizar flujos de fondos esperados provenientes del activo y pasivo de la firma. En el caso de traslaciones paralelas en la tasa de costos de capital de la firma y de la deuda, el concepto clásico de inmunización es efectivo, siendo la guía la duración. En otras palabras, se debe calzar las duraciones de flujos del activo y pasivo de la empresa a un horizonte común dado por la duration. Claro está, esto dependerá de la flexibilidad de los mismos, en otras palabras la posibilidad de postergar flujos de deuda o adelantar flujos de activos. En el caso de la firma, tal flexibilidad es factible en el caso de los flujos de pasivos, ya que la estructura temporal de los flujos de las inversiones si bien son gestionables por la gerencia, a menudo queda sujetas a las características operativas, tecnológicas y de mercado del negocio. Ahora bien, en el caso de traslaciones no paralelas en la tasas de costo de capital se debe buscar mayor convexidad generado por una mayor duración, caso que acontece en los activos de la firma producto del impacto en los flujos del valor terminal.

\section{REFERENCIAS BIBLIOGRÁFICAS}

Bradley, M-Gregg, J. (2008). Expected Inflation and The Constant Growth Valuation Model. Journal of Applied Corporate Finance, 20(2), 66-78.

Copeland, T -Koller, K -Murrin, J. (2000). Valuation: Measuring and Managing the Value of Companies (3 ed.). New York: Wiley.

Copeland, T- Weston, F- Shastri, K. (2004). Financial Theory and Corporate Policy (4 ed.). Estados Unidos: Pearson Addison Wesley.

Damodaran, A. (2015). Country Risk: Determinants, Measures and Implications The 2015 edition. (SSRN, Ed.) Social Science Research Network (SSRN), Downloads/SSRN-id2630871. pdf, 1-97.

Elton, D- Gruber, M. (1995). Modern Portfolio Theory and Investment Analysis (5 ed.). New York: John Wiley \& Sons.

Fabozzi, F-Fabozzi, D. (1996). Bond Markets, Analysis and Strategies. New Jersey, NJ: Prentice Hall. Englewood Cliffs. 
Fama, E-French, K. (1992). The cross-section of expected stock returns. Journal of Finance, $47,427-465$.

Fama, E-French, K. (2004). The capital asset pricing model: Theory and evidence. Journal of Economics Perspectives, 18(3), 25-46.

Fama, E-French, K. (1996). Multifactor explanations of asset pricing anomalies. Journal of Finance, 51, 55-84.

Fernández, P. (2014). Valoración de Empresas y Sensatez (Tercera ed.). Barcelona: IESE Business School-Universidad de Navarra.

Fornero, R. (2003). Finanzas de empresas en mercados emergentes. (S. A. Financiera, Ed.) Anales de las XXIII Jornadas de docentes en Administración Financiera SADAF, www.sadaf. com.ar/espanol/publicaciones/publicacion_individual.php?id=212, 107-125

Fornero, R. (2012). Análisis financiero e inflación. Mendoza, Argentina: Working Paper Universidad Nacional de Cuyo.

Jorion, P. (2003). Financial Risk Manager Handbook. New York: John Wiley \& Sons.

López Dumrauf, G. (2014). Análisis Cuantitativo de Bonos (1 ed.). Buenos Aires: Alfaomega.

Milanesi, G. (2017). Inflación y descuento de flujo de fondos en dos monedas. Un enfoque integral. Revista Argentina de Investigación en Negocios, 3(1), 89-109.

Pratt; S-Grabowski; R. (2008). Cost Of Capital: Applications and Examples (3 ed.). New Jersey: John Wiley \& Sons.

Sharpe, W. (1964). Capital asset prices: A theory of market equilibrium under conditions of risk. Journal of Finance, 19(3), 425-442.

Skinner, F. (2005). Pricing and Hedging Interest and Credit Risk Instrument. Oxford: Elsevier Finance. 


\section{CURRICULUM VITAE}

\section{Gstón Silverio Milanesi}

Doctor en Ciencias de la Administración (UNS), Magister en Administración (UNS), Contador Público (UNS), Profesor Titular Exclusivo Administración Financiera I y Decisiones y Estrategias Financieras carreras Contador Público y Licenciatura en Administración Departamento Ciencias de la Administración, UNS, Director Decano Departamento Ciencias de la Administración (UNS). milanesi@uns.edu.ar 